\title{
A simple recurrence formula for the number of rooted maps on surfaces by edges and genus.
}

\author{
Sean Carrel1 " $\|^{*}$ and Guillaume Chapuy $\|^{2}$ \\ ${ }^{1}$ Department of Combinatorics \& Optimization, University of Waterloo, Waterloo, Canada \\ ${ }^{2}$ CNRS and LIAFA, Université Paris Diderot-Paris 7, Paris, France
}

\begin{abstract}
We establish a simple recurrence formula for the number $Q_{g}^{n}$ of rooted orientable maps counted by edges and genus. The formula is a consequence of the KP equation for the generating function of bipartite maps, coupled with a Tutte equation, and it was apparently unnoticed before. It gives by far the fastest known way of computing these numbers, or the fixed-genus generating functions, especially for large $g$. The formula is similar in look to the one discovered by Goulden and Jackson for triangulations (although the latter does not rely on an additional Tutte equation). Both of them have a very combinatorial flavour, but finding a bijective interpretation is currently unsolved - should such an interpretation exist, the history of bijective methods for maps would tend to show that the case treated here is easier to start with than the one of triangulations.
\end{abstract}

Résumé. Nous établissons une formule de récurrence simple pour le nombre $Q_{n}^{g}$ de cartes enracinées de genre $g$ à $n$ arêtes. Cette formule est une conséquence relativement simple du fait que la série génératrice des cartes biparties est une solution de l'équation KP et d'une équation de Tutte, et elle était apparemment passée inaperçue jusque là. Elle donne de loin le moyen le plus rapide pour calculer ces nombres, en particulier quand $g$ est grand. La formule est d'apparence similaire à celle découverte par Goulden et Jackson pour les triangulations (quoique cette dernière ne repose pas sur une équation de Tutte additionnelle). Les deux formules ont une saveur très combinatoire, mais trouver une interprétation bijective reste un problème ouvert - mais si une telle interprétation existe, l'histoire des méthodes bijectives pour les cartes tendrait à montrer que le cas traité ici est plus facile pour commencer que celui des triangulations.

Keywords: Enumeration, maps on surfaces, quadrangulations, KP hierarchy

\section{Introduction and main formula}

A map is a connected graph embedded in a compact connected orientable surface in such a way that the regions delimited by the graph, called faces, are homeomorphic to open discs. Loops and multiple edges

\footnotetext{
*Email: s.r.carrellegmail.com.

${ }^{\dagger}$ Email: guillaume. chapuy@liafa.univ-paris-diderot.fr. Partial support from French Agence Nationale de la Recherche, grant number ANR 12-JS02-001-01 "Cartaplus", and from Ville de Paris, grant "Émergences Paris 2013, Combinatoire à Paris".
}

1365-8050 @ 2014 Discrete Mathematics and Theoretical Computer Science (DMTCS), Nancy, France 
are allowed. A rooted map is a map in which an angular sector incident to a vertex is distinguished, and the latter is called the root vertex. The root edge is the edge encountered when traversing the distinguished angular sector clockwise around the root vertex. Rooted maps are considered up to oriented homeomorphisms preserving the root sector.

A map is bipartite if its vertices can be coloured with two colors, say black and white, in such a way that each edge links a white and a black vertex. Unless otherwise mentioned, bipartite maps will be endowed with their canonical bicolouration in which the root vertex is coloured white. The degree of a face in a map is equal to the number of edge sides along its boundary, counted with multiplicity. Note that in a bipartite map every face has even degree, since colours alternate along its boundary.

A quadrangulation is a map in which every face has degree 4 . There is a classical bijection, that goes back to Tutte [20], between bipartite quadrangulations with $n$ faces and genus $g$, and rooted maps with $n$ edges and genus $g$. It is illustrated on Figure 1 .

For $g, n \geq 0$, we let $Q_{g}^{n}$ be the number of rooted bipartite quadrangulations of genus $g$ with $n$ faces. Equivalently, by Tutte's construction, $Q_{g}^{n}$ is the number of rooted maps of genus $g$ with $n$ edges. By convention we admit a single map with no edges and which has genus zero. Our main result is the following recurrence formula:

Theorem 1 The number $Q_{g}^{n}$ of rooted bipartite quadrangulations of genus $g$ with $n$ faces satisfies the following recurrence relation:

$\frac{n+1}{6} Q_{g}^{n}=\frac{4 n-2}{3} Q_{g}^{n-1}+\frac{(2 n-3)(2 n-2)(2 n-1)}{12} Q_{g-1}^{n-2}+\frac{1}{2} \sum_{\substack{k+\ell=n \\ k, \ell \geq 1}} \sum_{\substack{i+j=g \\ i, j \geq 0}}(2 k-1)(2 \ell-1) Q_{i}^{k-1} Q_{j}^{\ell-1}$,

for $n \geq 1$, with the initial conditions $Q_{0}^{0}=1$ and $Q_{g}^{0}=0$ for $g \geq 1$.

The rest of the paper is organized as follows. In Section 2, we prove Theorem 1 This result relies on both classical facts about the KP equation for bipartite maps, and an elementary Lemma obtained by combinatorial means (Lemma 3). In Section 3, we give a corollary of Theorem 1 in terms of generating functions (Theorem 47. In particular, we obtain a very efficient recurrence formula that can be used to compute the generating function of maps of fixed genus inductively. Finally, in Section 4 , we comment on the differences between what we do here and other known approaches to the problem: in brief, our method is much more powerful for the particular problem treated here, but we still don't know whether it can be applied successfully to cases other than bipartite quadrangulations.

\section{Proof of the main formula}

\subsection{Bipartite maps and KP equation}

The first element of our proof is the fact that the generating function for bipartite maps is a solution to the KP equation (Proposition 2 below). In the rest of the paper, the weight of a map is one over its number of edges, and a generating function of some family of maps is weighted if each map is counted with its weight in this generating function. We let $z, w$, and $\mathbf{p}=p_{1}, p_{2}, \ldots$ be infinitely many indeterminates. We extend the variables in $\mathbf{p}$ multiplicatively to partitions, i.e. we denote $p_{\alpha}:=\prod_{i} p_{\alpha_{i}}$ if $\alpha$ is a partition. The keystone of this paper is the following result ${ }^{(i)}$

\footnotetext{
(i) the literature on the KP hierarchy has been built over the years, with many references written by mathematical physicists and published in the physics literature. This is especially true for the link with map enumeration, often arising in formal expansions of
} 




(a) A map m

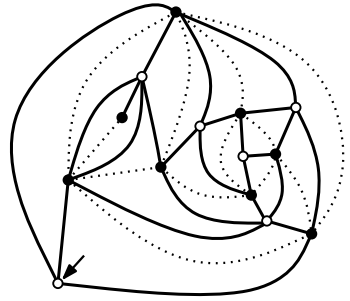

(b) its associated bipartite quadrangulation $\mathfrak{q}$ (thick edges)

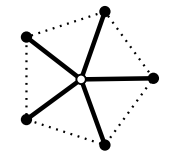

(c) the local rules of the construction around a face of $\mathfrak{m}$

Fig. 1: Tutte's bijection. Given a (not necessarily bipartite) map $\mathfrak{m}$ of genus $g$ with $n$ edges, add a new (white) vertex inside each face of $\mathfrak{m}$, and link it by a new edge to each of the corners incident to the face. The bipartite quadrangulation $\mathfrak{q}$ is obtained by erasing all the original edges of $\mathfrak{m}$, i.e. by keeping only the new (white) vertices, the old (black) vertices, and the newly created edges. The root edge of $\mathfrak{q}$ is the one created from the root corner of $\mathfrak{m}$ (which is enough to root $\mathfrak{q}$ if we demand that its root vertex is white). (a) and (b) display an example of the construction for a map of genus 0 (embedded on the sphere). Root corners are indicated by arrows.

Proposition 2 ([13], see also [18] ) For $n, v \geq 1$, and $\alpha \vdash n$ a partition of $n$, let $H_{\alpha}(n, v)$ be the number of rooted bipartite maps with $n$ edges and $v$ vertices, where the half face degrees are given by the parts of $\alpha$. Let $H=H(z, w ; \mathbf{p})$ be the weighted generating function of bipartite maps, with $z$ marking edges, $w$ marking vertices, and the $p_{i}$ marking the number of faces of degree $2 i$ for $i \geq 1$ :

$$
H(z, w ; \mathbf{p}):=1+\sum_{\substack{n \geq 1 \\ v \geq 1}} \frac{w^{v} z^{n}}{n} \sum_{\alpha \vdash n} H_{\alpha}(n, v) p_{\alpha} .
$$

Then $H$ is a solution of the KP equation:

$$
-H_{3,1}+H_{2,2}+\frac{1}{12} H_{1^{4}}+\frac{1}{2}\left(H_{1,1}\right)^{2}=0
$$

where indices indicate partial derivatives with respect to the variables $p_{i}$, for example $H_{3,1}:=\frac{\partial^{2}}{\partial p_{3} \partial p_{1}} H$. Actually, the generating function $H$ is a solution of an infinite system of partial differential equations, known as the KP Hierarchy (see, e.g., [16, 13, 4]), but we will need only the simplest one of these equations here, namely [1].

Proof: First recall that a bipartite map $\mathfrak{m}$ with $n$ edges labelled from 1 to $n$ can be encoded by a triple of permutations $\left(\sigma_{\circ}, \sigma_{\bullet}, \phi\right) \in\left(S_{n}\right)^{3}$ such that $\sigma_{\circ} \sigma_{\bullet}=\phi$. In this correspondence, the cycles of the permutation $\sigma_{\circ}$ (resp. $\sigma_{\bullet}$ ) encode the counterclockwise ordering of the edges around the white (resp.

matrix integrals. Thus it is not always easy for the mathematician to know who to attribute the results in this field. The reader may consult [15. Chapter 5] for historical references related to matrix integrals in the physics literature, and [13 4] for self-contained proofs written in the language of algebraic combinatorics. As for Proposition 2 it is essentially a consequence of the classical fact that map generating functions can be written in terms of Schur functions (see e.g. [14]), together with a result of Orlov and Shcherbin [18] that imply that certain infinite linear combinations of Schur functions satisfy the KP hierarchy. To be self-contained here, we have chosen to give the most easily checkable reference, and we prove Proposition 2 by giving all the details necessary to make the link with an equivalent statement in [13]. 
black) vertices of $\mathfrak{m}$, while the cycles of $\phi$ encode the clockwise ordering of the white to black edge-sides around the faces of $\mathfrak{m}$. This encoding gives a 1 to $(n-1)$ ! correspondence between rooted bipartite maps with $n$ edges and triples of permutations as above that are transitive, i.e. that generate a transitive subgroup of $S_{n}$. We refer to [9], or Figure 2 for more about this encoding (see also [15, 14]).

Now recall Theorem 3.1 in [13]. Let $b_{\alpha, \beta}^{\left(a_{1}, a_{2}, \cdots\right)}$ be the number of tuples of permutations $\left(\sigma, \gamma, \pi_{1}, \pi_{2}, \cdots\right)$ on $\{1, \cdots, n\}$ such that

1. $\sigma$ has cycle type $\alpha, \gamma$ has cycle type $\beta$ and $\pi_{i}$ has $n-a_{i}$ cycles for each $i \geq 1$;

2. $\sigma \gamma \pi_{1} \pi_{2} \cdots=1$ in $S_{n}$ where 1 is the identity;

3. the subgroup generated by $\sigma, \gamma, \pi_{1}, \pi_{2}, \cdots$ acts transitively on $\{1, \cdots, n\}$. Then the series

$$
B=\sum_{\substack{|\alpha|=|\beta|=n \geq 1, a_{1}, a_{2}, \cdots \geq 0}} \frac{1}{n !} b_{\alpha, \beta}^{\left(a_{1}, a_{2}, \cdots\right)} p_{\alpha} q_{\beta} u_{1}^{a_{1}} u_{2}^{a_{2}} \ldots
$$

is a solution to the KP hierarchy in the variables $p_{1}, p_{2}, \ldots$. Here $q_{1}, q_{2}, \ldots$ and $u_{1}, u_{2}, \ldots$ are two infinite sets of auxiliary variables, and we use the notation $q_{\beta}=\prod_{i} q_{\beta_{i}}$.

Now, using the encoding of maps as triples of permutations as above, we see that $(n-1) ! H_{\alpha}(n, v)=$ $\sum_{i \geq 0} b_{\alpha, 1^{n}}^{(n-i, n+i-v, 0, \cdots)}$,since the coefficient on the right hand side is the number of solutions to the equation $\sigma \gamma \pi_{1} \pi_{2}=1$ where the total number of cycles in $\pi_{1}$ and $\pi_{2}$ is $v, \sigma$ has cycle type $\alpha$ and where $\gamma$ is the identity. Multiplying by $\sigma^{-1}$ then gives $\pi_{1} \pi_{2}=\sigma^{-1}$ which matches the encoding of bipartite maps given above. Thus, by setting $q_{1}=w^{2} z, q_{i}=0$ for $i \geq 2, u_{1}=u_{2}=w^{-1}$ and $u_{i}=0$ for $i \geq 3$ in $B$, we get the series $H$ as required.

Note that we choose to attribute this result to [13] since this provides a clear and checkable mathematical reference. The result was refered to before this reference in the mathematical physics literature, however, it is hard to find references in which the result is properly stated or proved. We refer to Chapter 5 of the book [15] as an entry point for the interested reader.

\subsection{Bipartite quadrangulations}

Our goal is to use Proposition 2 to get information on the generating function of bipartite quadrangulations. To this end, we let $\theta$ denote the operator that substitutes the variable $p_{2}$ to 1 and all the variables $p_{i}$

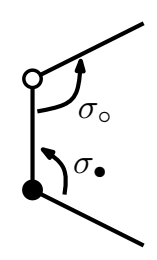

(a)

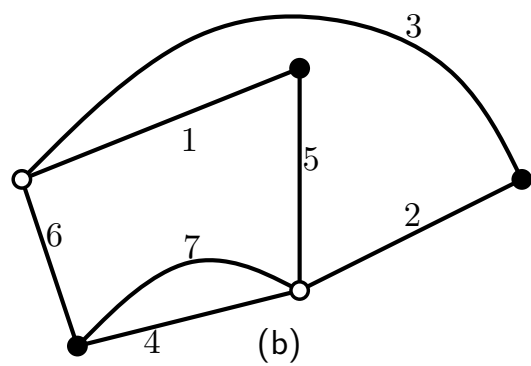

(b)

$$
\begin{aligned}
\sigma_{\circ} & =(1,3,6)(2,5,7,4) \\
\sigma_{\bullet} & =(1,5)(2,3)(4,7,6) \\
\sigma_{\circ} \sigma_{\bullet} & =(1,7)(2,6)(3,5)(4)
\end{aligned}
$$

(c)

Fig. 2: (a) The rules defining the permutations $\sigma_{\circ}$ and $\sigma_{\bullet}$. (b) A bipartite map with 7 edges arbitrarily labelled from 1 to 7 . (c) The corresponding permutations $\sigma_{\circ}$ and $\sigma_{\bullet}$. 
to 0 for $i \neq 2$. When we apply $\theta$ to 11 we get four terms:

$$
-\theta H_{3,1}+\theta H_{2,2}+\frac{1}{12} \theta H_{1^{4}}+\frac{1}{2}\left(\theta H_{1,1}\right)^{2}=0 .
$$

Note that since all the derivatives appearing in (1) are with respect to $p_{1}, p_{2}$ or $p_{3}$, any monomial in $H$ that contains a variable $p_{i}$ for some $i \neq\{1,2,3\}$ gives a zero contribution to (2). Therefore each of the four terms appearing in (2) can be interpreted as the generating function of some family of bipartite maps having only faces of degree 2,4 , or 6 (subject to further restrictions). However, thanks to local operations on maps, we will be able to relate each term to maps having only faces of degree 4 , as shown by the next lemma.

If $A(z, w)$ is a formal power series in $z$ and $w$ we denote by $\left[z^{p} w^{q}\right] A(z, w)$ the coefficient of the mononial $z^{p} w^{q}$ in $A(z, w)$.

Lemma 3 Let $n, g \geq 1$. Then we have:

$$
\begin{aligned}
{\left[z^{2 n} w^{n+2-2 g}\right] \theta H_{2,2} } & =\frac{n-1}{2} Q_{g}^{n}, \\
{\left[z^{2 n} w^{n+1-2 g}\right] \theta H_{1,1} } & =(2 n-1) Q_{g}^{n-1}, \\
{\left[z^{2 n} w^{n+2-2 g}\right] \theta H_{1^{4}} } & =(2 n-1)(2 n-2)(2 n-3) Q_{g-1}^{n-2}, \\
{\left[z^{2 n} w^{n+2-2 g}\right] \theta H_{3,1} } & =\frac{2 n-1}{3}\left(Q_{g}^{n}-2 Q_{g}^{n-1}\right) .
\end{aligned}
$$

We now prove the lemma. By definition, if $v \geq 1$ and $\lambda=\left(\lambda_{1}, \lambda_{2}, \ldots, \lambda_{\ell}\right)$ is a partition of some integer, then $\left[z^{2 n} w^{v}\right] \theta H_{\lambda}$ is $\frac{1}{2 n}$ times the number of rooted bipartite maps with $2 n$ edges, $v$ vertices, $\ell$ marked (numbered) faces of degrees $2 \lambda_{1}, 2 \lambda_{2}, \ldots, 2 \lambda_{\ell}$, and all other (unmarked) faces of degree 4 . If $r$ is the number of unmarked faces, such a map has $r+\ell$ faces, and by Euler's formula, the genus $g$ of this map satisfies: $v-2 n+(r+\ell)=2-2 g$. Moreover the number of edges is equal to the sum of the half face degrees so $2 n=2 r+|\lambda|$, therefore we obtain the relation:

$$
2 g=n+2-v+\frac{|\lambda|}{2}-\ell
$$

which we shall use repeatedly. We now proceed with the proof of Lemma 3 .

Proof of (3): As discussed above, $H_{2,2}$ is the weighted generating function of rooted bipartite maps with two marked faces of degree 4 , so $\theta H_{2,2}$ is the weighted generating function of rooted quadrangulations with two marked faces. Moreover, by (7), the maps that contribute to the coefficient $\left[z^{2 n} w^{n+2-2 g}\right]$ in $\theta H_{2,2}$ have genus $g$. Now, there are $n(n-1)$ ways of marking two faces in a quadrangulation with $n$ faces, and the weight of such a map is $\frac{1}{2 n}$ since it has $2 n$ edges. Therefore: $\left[z^{2 n} w^{n+2-2 g}\right] \theta H_{2,2}=$ $\frac{1}{2 n} n \cdot(n-1) Q_{g}^{n}$.

Proof of (4) and (5): As discussed above, for $k \geq 1, \theta H_{1^{2 k}}$ is the weighted generating function of bipartite maps carrying $2 k$ marked (numbered) faces of degree 2 , having all other faces of degree 4 . Moreover, by (7), the genus of maps that contribute to the coefficient $\left[z^{2 n} w^{n+k-2 g}\right]$ in this series is equal to $g+1-k$. Therefore:

$$
\left[z^{2 n} w^{n+k-2 g}\right] \theta H_{1^{2 k}}=\frac{1}{2 n} P_{g+1-k}^{2 n, 2 k}
$$


where $P_{h}^{m, \ell}$ denotes the number of rooted bipartite maps of genus $h$ with $\ell$ numbered marked faces of degree 2 , all other faces of degree 4 , and $m$ edges in total. Now, we claim that for all $h$ and all $m, \ell$ with $m+\ell$ even one has:

$$
P_{h}^{m, \ell}=m(m-1) \ldots(m-\ell+1) Q_{h}^{\frac{m-\ell}{2}} .
$$

This is obvious for $\ell=0$ since a quadrangulation with $m$ edges has $m / 2$ faces. For $\ell \geq 1$, consider a bipartite map with all faces of degree 4 , except $\ell$ marked faces of degree 2 , and $m$ edges in total. By contracting the first marked face into an edge, one obtains a map with one less marked face, and a marked edge. This marked edge can be considered as the root edge of that map (keeping the canonical bicolouration of vertices). Conversely, starting with a map having $\ell-1$ marked faces, and $m-1$ edges, and expanding the root-edge into a face of degree 2, there are $m$ ways of choosing a root corner in the resulting map in a way that preserves the canonical bicolouration of vertices. Therefore one has $P_{h}^{m, \ell}=m \cdot P_{h}^{m-1, \ell-1}$ and $(9)$ follows by induction. (4) and (5) then follows from (8) for $k=1$ and $k=2$, respectively.

Proof of (6): This case starts in the same way as the three others, but we will have to use an additional tool (a simple Tutte equation) in order to express everything in terms of quadrangulation numbers only. First, $\theta H_{3,1}$ is the weighted generating function of rooted bipartite maps with one face of degree 6 , one face of degree 2, and all other faces of degree 4 . Moreover, by (7), maps that contribute to the coefficient of $\left[z^{2 n} w^{n+2-2 g}\right]$ in this series all have genus $g$. We first get rid of the face of degree 2 by contracting it into an edge, and declare this edge as the root of the new map, keeping the canonical bicolouration. If the original map has $2 n$ edges, we obtain a map with $2 n-1$ edges in total. Conversely, if we start with a map with $2 n-1$ edges and we expand the root edge into a face of degree 2 , we have $2 n$ ways of choosing a new root corner in the newly created map, keeping the canonical bicolouration. Therefore if we let $X_{g}^{n}$ be the number of rooted bipartite maps having a face of degree 6 , all other faces of degree 4 , and $2 n-1$ edges in total, we have:

$$
\left[z^{2 n} w^{n+2-2 g}\right] \theta H_{3,1}=\frac{1}{2 n} \cdot 2 n X_{g}^{n}=X_{g}^{n},
$$

where the first factor is the weight coming from the definition of $H$. Thus to prove (6) it is enough to establish the following equation:

$$
Q_{g}^{n}=\frac{3}{2 n-1} X_{g}^{n}+2 Q_{g}^{n-1} .
$$

The reader well acquainted with map enumeration may have recognized in 10 a (very simple case of a) Tutte/loop equation. It is proved as follows. Let $\mathfrak{q}$ be a rooted bipartite quadrangulation of genus $g$ with $n$ faces, and let $e$ be the root edge of $\mathfrak{q}$. There are two cases: 1. the edge $e$ is bordered by two distinct faces, and 2. the edge $e$ is bordered twice by the same face.

In case 1., removing the edge $e$ gives rise to a map of genus $g$ with a marked face of degree 6 . By marking one of the $2 n-1$ white corners of this map as the root, we obtain a rooted map counted by $X_{g}^{n}$, and since there are 3 ways of placing a diagonal in a face of degree 6 to create two quadrangles, the counting number $N_{1}$ corresponding to case 1 . satisfies $(2 n-1) N_{1}=3 X_{g}^{n}$.

In case 2., the removal of the edge $e$ creates two faces (a priori, either in the same or in two different connected components) of degrees $k_{1}, k_{2}$ with $k_{1}+k_{2}+2=4$. Now since $\mathfrak{q}$ is bipartite, $k_{1}$ and $k_{2}$ are even 
which shows that one of the $k_{i}$ is zero and the other is equal to 2 . Therefore, in $\mathfrak{q}, e$ is a single edge hanging in a face of degree 2. By removing $e$ and contracting the degree 2 face, we obtain a quadrangulation with $n-1$ faces (and a marked edge that serves as a root, keeping the canonical bicolouration). Since there are two ways to attach a hanging edge in a face of degree 2 , the counting number corresponding to case 2 . is $N_{2}=2 Q_{g}^{n-1}$.

Writing that $Q_{g}^{n}=N_{1}+N_{2}$, we obtain 10 and complete the proof.

Proof of Theorem 1: Just extract the coefficient of $\left[z^{n} w^{n+2-2 g}\right]$ in Equation (2) using Lemma 3, and group together the two terms containing $Q_{g}^{n}$, namely $\frac{n-1}{2} Q_{g}^{n}-\frac{2 n-1}{3} Q_{g}^{n}=-\frac{n+1}{6} Q_{g}^{n}$.

\section{Fixed genus generating functions}

Let $Q_{g}(t):=\sum_{n>0} Q_{g}^{n} t^{n}$ be the generating function of rooted maps of genus $g$ by the number of edges. It was shown in [2] that $Q_{g}(t)$ is a rational function of $\rho:=\sqrt{1-12 t}$. In genus 0 , the result goes back to Tutte [20] and one has the explicit expression:

$$
Q_{0}(t)=T-t T^{3}
$$

where $T=\frac{1-\sqrt{1-12 t}}{6 t}$ is the unique formal power series solution of the equation

$$
T=1+3 t T^{2}
$$

In the following we will give a very simple recursive formula to compute the series $Q_{g}(t)$ as a rational function of $T$, and we will study some of its properties ${ }^{\text {(ii) }}$

Theorem 4 For $g \geq 0$, we have $Q_{g}(t)=R_{g}(T)$ where $T$ is given by (12) and $R_{g}$ is a rational function that can be computed iteratively via:

$$
\begin{aligned}
& \frac{d}{d T}\left(\frac{(T-1)(T+2)}{3 T} R_{g}(T)\right) \\
= & \frac{(T-1)^{2}}{18 T^{4}}(2 D+1)(2 D+2)(2 D+3) R_{g-1}(T)+\frac{(T-1)^{2}}{3 T^{4}} \sum_{\substack{i+j=g \\
i, j \geq 1}}\left((2 D+1) R_{i}(T)\right)\left((2 D+1) R_{j}(T)\right)
\end{aligned}
$$

where $D=\frac{T(1-T)}{T-2} \frac{d}{d T}$.

(ii) Note that being a rational function of $T$ or $\rho$ is equivalent, but we prefer to work with $T$, since as a power series $T$ has a clear combinatorial meaning. Indeed, $T$ is the generating function of labelled/blossomed trees, which are the fundamental building blocks that underly the bijective decomposition of maps [19 8, 7]. It is thus tempting to believe that those rationality results have a combinatorial interpretation in terms of these trees, even if it is still an open problem to find one. Indeed, so far the best rationality statement that is understood combinatorially is that the series of rooted bipartite quadrangulations of genus $g$ with a distinguished vertex is a rational function in the variable $U$ such that $1=t T^{2}\left(1+U+U^{-1}\right)$, which is weaker than the rationality in $T$. See [7] for this result. 
Proof: First, one easily checks that Theorem 1 is equivalent to the following differential equation:

$$
\begin{aligned}
& (D+1) Q_{g}= \\
& 4 t(2 D+1) Q_{g}+\frac{1}{2} t^{2}(2 D+1)(2 D+2)(2 D+3) Q_{g-1}+3 t^{2} \sum_{\substack{i+j=g \\
i, j \geq 0}}\left((2 D+1) Q_{i}\right)\left((2 D+1) Q_{j}\right),
\end{aligned}
$$

where $D$ is the operator $D:=t \cdot \frac{d}{d t}$. Using (12) one checks that $T^{\prime}(t)=\frac{T(1-T)}{T-2}$, so that $D=$ $\left(\frac{d T(t)}{d t}\right) \frac{d}{d T}=\frac{T(1-T)}{T-2} \frac{d}{d T}$ and the definition of $D$ coincides with the one given in the statement of the theorem.

Now, for $h \geq 0$ let $R_{h}$ be the unique formal power series such that $Q_{h}(t)=R_{h}(T)$. Grouping all the genus $g$ generating functions on the left hand side, we can put (14) in the form:

$$
A R_{g}(t)+B \frac{d}{d T} R_{g}(t)=\text { R.H.S. }
$$

where $\left.A=1-4 t-6 t^{2}(2 D+1) Q_{0}, B=t\left(1-8 t-12 t^{2}(2 D+1) Q_{0}\right)\right)$, and the R.H.S. is the same as in (13). Using the explicit expression (11) of $Q_{0}$ in terms of $T$, we can then rewrite the L.H.S. of (15) as

$$
\frac{(T-1)(T+2)}{3 T} R_{g}^{\prime}(T)+\frac{T^{2}+2}{3 T^{2}} R_{g}(T)=\frac{d}{d T}\left(\frac{(T-1)(T+2)}{3 T} R_{g}(T)\right),
$$

and we obtain $(13)$. Note that we have not proved that $R_{g}(T)$ is a rational function: we admit this fact from [2].

Observe that we have $R_{g}(1)=Q_{g}(0)<\infty$ so the quantity $\frac{(T-1)(T+2)}{3 T} R_{g}(T)$ vanishes at $T=1$, and we have:

$$
\frac{(T-1)(T+2)}{3 T} R_{g}(T)=\int_{1}^{T} \text { R.H.S. }
$$

with the R.H.S. given by (13), which shows that (13) indeed enables one to compute the $R_{g}$ 's recursively. Note that it is not obvious a priori that no logarithm appears during this integration, although this is true since it is known that $R_{g}$ is rationa (iii) [2]. Moreover, since all generating functions considered are finite at $T=1$ (which corresponds to the point $t=0$ ) we obtain via an easy induction that $R_{g}$ has only poles at $T=2$ or $T=-2$. More precisely, by an easy induction, we obtain a bound on the degrees of the poles:

Corollary 5 For $g \geq 1$ we have $Q_{g}(t)=R_{g}(T)$ where $R_{g}$ can be written as:

$$
R_{g}=c_{0}^{(g)}+\sum_{i=1}^{5 g-3} \frac{\alpha_{i}^{(g)}}{(2-T)^{i}}+\sum_{i=1}^{3 g-2} \frac{\beta_{i}^{(g)}}{(T+2)^{i}}
$$

for rational numbers $c_{0}^{(g)}$ and $\alpha_{i}^{(g)}, \beta_{i}^{(g)}$.

Note that by plugging the ansatz (16) into the recursion (13), we obtain a very efficient way of computing the $R_{g}$ 's inductively.

(iii) We unfortunately haven't been able to reprove this fact from our approach 
We conclude this section with (known) considerations on asymptotics. From (16), it is easy to see that the dominant singularity of $Q_{g}(t)$ is unique, and is reached at $t=\frac{1}{12}$, i.e. when $T=2$. In particular the dominant term in 16 is $\frac{\alpha_{5 g-3}^{(g)}}{(2-T)^{5 g-3}}$. Using the fact that $2-T=2 \sqrt{1-12 t}+O(1-12 t)$ when $t$ tends to $\frac{1}{12}$, and using a standard transfer theorem for algebraic functions [11], we obtain that for fixed $g$, $n$ tending to infinity:

$$
Q_{g}^{n} \sim t_{g} n^{\frac{5(g-1)}{2}} 12^{n}
$$

with $t_{g}=\frac{1}{2^{5 g-3} \Gamma\left(\frac{5 g-3}{2}\right)} \alpha_{5 g-3}^{(g)}$. Moreover, by extracting the leading order coefficient in 13 when $T \sim 2$, we see with a short computation that the sequence $\tau_{g}=(5 g-3) \alpha_{5 g-3}^{(g)}=2^{5 g-2} \Gamma\left(\frac{5 g-1}{2}\right) t_{g}$ satisfies the following Painlevé-I type recursion

$$
\tau_{g}=\frac{1}{3}(5 g-4)(5 g-6) \tau_{g-1}+\frac{1}{2} \sum_{h=1}^{g-1} \tau_{h} \tau_{g-h}
$$

which enables one to compute the $t_{g}$ 's easily by induction starting from $t_{1}=\frac{1}{24}$ (i.e. $\tau_{1}=\frac{1}{3}$ ). These results are well known (for (17) see [1]; for [18] see [15, p.201], or [3]). So far, as far as we know, all the known proofs of 18 rely on integrable hierarchies.

\section{Discussion and comparison with other approaches}

In this paper we have obtained a simple recurrence formula to compute the numbers $Q_{g}^{n}$ of rooted maps of genus $g$ with $n$ edges inductively. It gives rise to a very efficient inductive formula to compute the fixed genus generating functions. Let us now compare with other existing approaches to enumerate maps on surfaces.

Tutte/loop equations. The most direct way to count maps on surfaces is to perform a root edge decomposition, whose counting counterpart is known as Tutte equation (or loop equation in the context of mathematical physics). This approach enabled Bender and Canfield [2] to prove the rationality of the generating function of maps in terms of the parameter $\rho$ as discussed in Section 3, and was generalized to other classes of maps via variants of the kernel method (see, e.g., [12]). This approach has been considerably improved by the Eynard school (see e.g. [10]) who developed powerful machinery to solve recursively these equations for many families of maps.

However, because they are based on Tutte equations, both the methods of [2, 12] and [10] require working with generating functions of maps carrying an arbitrarily large number of additional boundaries. To illustrate, in the special case of quadrangulations, the "topological recursions" given by these papers enable one to compute inductively the generating functions $Q_{g}^{(p)}(t) \equiv Q_{g}\left(t ; x_{1}, x_{2}, \ldots, x_{p}\right)$ of rooted quadrangulations of genus $g$ carrying $p$ additional faces of arbitrary degree, marked by the additional variables $x_{1}, x_{2}, \ldots, x_{p}$. In order to be able to compute $Q_{g}^{(p)}(t)$ these recursions take as an input the planar generating function $Q_{0}^{(g+k)}(t)$, so one cannot avoid working with these extra variables (linearly many of them with respect to the genus), even to compute the pure quadrangulation series $Q_{g}^{(0)}$.

Compared to this, the recurrence relations obtained in this paper (Theorems 1 and 4 are much more efficient, as they require only two variables ( $n$ and $g$, or $t$ and $g$ ). In particular we can compute all generating functions easily, for large $g$, the main limit being the size of the output. However, of course, what we 
do here is a very special case: we consider only bipartite quadrangulations, whereas the aforementioned approaches enable one to count maps with arbitrary degree distribution!

Integrable hierarchies. It has been known for some time in the context of mathematical physics that multivariate generating functions of maps are solution of integrable hierarchies of partial differential equations such as the KP or the Toda hierarchy, see e.g. [17, 18, 15, 13]. However these hierarchies do not characterize their solutions (as shown by the fact that many combinatorial models give different solutions), and one needs to add extra information to compute the generating functions. We know of at least three situations in which this is possible. The first one is Okounkov's work on Hurwitz numbers [17], where the integrable hierarchy is the 2-Toda hierarchy, and the "extra information" takes the form of the computation of a commutator of operators in the infinite wedge space [17, section 2.7].

The second one is Goulden and Jackson's recurrence for triangulations [13, Theorem 5.4], which looks very similar to our main result. The starting equation is the same as ours (Equation (1)), but for the generating function of ordinary (non bipartite) maps. In order to derive a closed equation from it, the authors of [13] do complicated manipulations of generating functions, but what they do could equivalently be done via local manipulations similar to the ones we used in the proofs of (4), (3), (5). We leave as an exercise to the reader the task of reproving [13, Theorem 5.4] along these lines (and with almost no computation).

The last one is the present paper, where in addition to such local manipulations, we use an additional, very degenerate, Tutte equation (Equation (10)). It seems difficult to find other cases than triangulations and bipartite quadrangulations where the same techniques would apply, even by allowing the use of more complicated Tutte equations. In our current understanding, this situation is a bit mysterious to us.

To conclude on this aspect, let us observe that the equations obtained from integrable hierarchies rely on the deep algebraic structure of the multivariate generating series of combinatorial maps (and on their link with Schur functions). This structure provides them with many symmetries that are not apparent in the combinatorial world, and we are far from understanding combinatorially the meaning of these equations. In particular, to our knowledge, the approaches based on integrable hierarchies are the only ones that enable one to prove statements such as $[18$.

Bijective methods. In the planar case $(g=0)$ the combinatorial structure of maps is now well understood thanks to bijections that relate maps to some kinds of decorated trees. The topic was initiated by Schaeffer [19, 8] and has been developped by many others. For these approaches, the simplest case turns out to be the one of bipartite quadrangulations. In this case, the trees underlying the bijective decompositions have a generating function given by 12 .

The bijective combinatorics of maps on other orientable surfaces is a more recent topic. Using bijections similar to the ones in the planar case, one can prove bijectively rationality results for the fixed-genus generating function of quadrangulations [7] or more generally fixed degree bipartite maps or constellations [5]. However, with these techniques, one obtains rationality in terms of some auxiliary generating functions whose degree of algebraicity is in general too high compared to the known non-bijective result. See the footnote page 579 for an example of this phenomenon in the case of quadrangulations. Moreover, although the asymptotic form [17) is well explained by these methods [7, 5, 6], they do not provide any information on the numbers $t_{g}$, and do not explain the relation 18 .

Therefore we are still far from being able to prove an exact counting statement such as Theorem 1 combinatorially. However, the history of bijective methods for maps tells us two things. First, that when a bijective approach exists to some map counting problem, the case of bipartite quadrangulations is always 
the easiest one to start with. Second, that before trying to find bijections, it is important to know what to prove bijectively. Therefore we hope that, in years to come, Theorem 1 will play a role guiding new developments of the bijective approaches to maps on surfaces.

\section{References}

[1] Edward A. Bender and E. Rodney Canfield. The asymptotic number of rooted maps on a surface. $J$. Combin. Theory Ser. A, 43(2):244-257, 1986.

[2] Edward A. Bender and E. Rodney Canfield. The number of rooted maps on an orientable surface. $J$. Combin. Theory Ser. B, 53(2):293-299, 1991.

[3] Edward A. Bender, Zhicheng Gao, and L. Bruce Richmond. The map asymptotics constant $t_{g}$. Electron. J. Combin., 15(1):Research paper 51, 8, 2008.

[4] S. R. Carrell and I. P. Goulden. Symmetric functions, codes of partitions and the KP hierarchy. $J$. Algebraic Combin., 32(2):211-226, 2010.

[5] Guillaume Chapuy. Asymptotic enumeration of constellations and related families of maps on orientable surfaces. Combin. Probab. Comput., 18(4):477-516, 2009.

[6] Guillaume Chapuy. The structure of unicellular maps, and a connection between maps of positive genus and planar labelled trees. Probab. Theory Related Fields, 147(3-4):415-447, 2010.

[7] Guillaume Chapuy, Michel Marcus, and Gilles Schaeffer. A bijection for rooted maps on orientable surfaces. SIAM J. Discrete Math., 23(3):1587-1611, 2009.

[8] Philippe Chassaing and Gilles Schaeffer. Random planar lattices and integrated superBrownian excursion. Probab. Theory Related Fields, 128(2):161-212, 2004.

[9] Robert Cori and Antonio Machì. Maps, hypermaps and their automorphisms: a survey. I, II, III. Exposition. Math., 10(5):403-427, 429-447, 449-467, 1992.

[10] Bertrand Eynard. Counting Surfaces. Book in preparation, available on the author's webpage.

[11] Philippe Flajolet and Andrew Odlyzko. Singularity analysis of generating functions. SIAM J. Discrete Math., 3(2):216-240, 1990.

[12] Zhicheng Gao. The number of degree restricted maps on general surfaces. Discrete Math., 123(13):47-63, 1993.

[13] I. P. Goulden and D. M. Jackson. The KP hierarchy, branched covers, and triangulations. Adv. Math., 219(3):932-951, 2008.

[14] D. M. Jackson and T. I. Visentin. A character-theoretic approach to embeddings of rooted maps in an orientable surface of given genus. Trans. Amer. Math. Soc., 322(1):343-363, 1990.

[15] Sergei K. Lando and Alexander K. Zvonkin. Graphs on surfaces and their applications, volume 141 of Encyclopaedia of Mathematical Sciences. Springer-Verlag, Berlin, 2004. With an appendix by Don B. Zagier, Low-Dimensional Topology, II. 
[16] T. Miwa, M. Jimbo, and E. Date. Solitons, volume 135 of Cambridge Tracts in Mathematics. Cambridge University Press, Cambridge, 2000. Differential equations, symmetries and infinitedimensional algebras, Translated from the 1993 Japanese original by Miles Reid.

[17] Andrei Okounkov. Toda equations for Hurwitz numbers. Math. Res. Lett., 7(4):447-453, 2000.

[18] A. Yu. Orlov and D. M. Shcherbin. Hypergeometric solutions of soliton equations. Teoret. Mat. Fiz., 128(1):84-108, 2001.

[19] Gilles Schaeffer. Bijective census and random generation of Eulerian planar maps with prescribed vertex degrees. Electron. J. Combin., 4(1):Research Paper 20, 14 pp. (electronic), 1997.

[20] W. T. Tutte. A census of planar maps. Canad. J. Math., 15:249-271, 1963. 